\section{BMJ Global Health}

\title{
I do what a woman should do': a grounded theory study of women's menstrual experiences at work in Mukono District, Uganda
}

\author{
Julie Hennegan (D) , ${ }^{1}$ Simon P S Kibira (D) ,2 Natalie G Exum (D) , \\ Kellogg J Schwab, ${ }^{1}$ Fredrick E Makumbi, ${ }^{3}$ Justine Bukenya ${ }^{2}$
}

To cite: Hennegan J, Kibira SPS, Exum NG, et al. I do what a woman should do': a grounded theory study of women's menstrual experiences at work in Mukono District, Uganda. BMJ Global Health 2020;5:e003433. doi:10.1136/ bmjgh-2020-003433

Handling editor Kerry Scott

- Additional material is published online only. To view, please visit the journal online (http://dx.doi.org/10.1136/ bmjgh-2020-003433).

Received 13 July 2020 Revised 14 0ctober 2020 Accepted 16 October 2020

Check for updates

\section{(c) Author(s) (or their} employer(s)) 2020. Re-use permitted under CC BY-NC. No commercial re-use. See rights and permissions. Published by BMJ.

For numbered affiliations see end of article.

\section{Correspondence to} Dr Julie Hennegan; jhenneg1@jhu.edu

\section{ABSTRACT}

Menstrual health has received increasing recognition as an essential issue for public health and gender equality. A growing body of research has elucidated adolescent girls' menstrual needs and informed policy and practice responses. However, the experiences of adult women have received little attention, particularly in the workplace where many spend a significant proportion of their lives. To address this gap, we took a grounded theory approach to generate a nuanced understanding of working women's menstrual experiences, and the impact of menstruation on their work and health in Mukono District, Uganda. In-depth interviews were undertaken with 35 women aged 18-49. This included 21 women working in markets, 7 teachers and 7 healthcare facility workers. Frequent collaborative analysis sessions throughout data collection, coding of interview transcripts, and generation of participant, workplace, and category memos facilitated analysis. Our core category and underlying theory, being a responsible woman', underpinned women's experiences. Being responsible' meant keeping menstruation secret, and the body clean, at all times. These gendered expectations meant that any difficulty managing menses represented a failure of womanhood, met with disgust and shame. Difficulties with menstrual pain and heavy bleeding were excepted from these expectations and perceived as requiring compassion. Commercial menstrual products were expensive for most women, and many expressed concerns about the quality of cheaper brands. Workplace infrastructure, particularly unreliable water supply and cleanliness, was problematic for many women who resorted to travelling home or to other facilities to meet their needs. Menstruation presented a burden at work, causing some women to miss work and income, and many others to endure pain, discomfort and anxiety throughout their day. Our findings can inform norm and resource-focused responses to improve experiences and should provoke critical reflection on the discourse used in menstrual health advocacy in Uganda.

\section{INTRODUCTION}

Half the population will experience a menstrual cycle for up to 40 years of their life.

\section{Key questions}

What is already known?

- Increased policy and practice aim to improve menstrual health; however, these efforts have focused almost exclusively on adolescent girls.

- Across a small number of qualitative studies, adult women have reported negative experiences of menstruation at home.

- There is little research evidence to understand women's menstrual experiences at work, and the impact of menstruation on health and well-being at work.

\section{What are the new findings?}

- Upholding gendered ideals of being a responsible woman during menstruation places women under significant pressure to keep the body clean and menstruation hidden at work.

- Difficulties managing menstrual bleeding, pain and discomforts associated with menstruation resulted in negative impacts on women's financial, social, mental and physical well-being at work.

- Many women reported poor performance and irritation associated with some disposable menstrual pad brands.

\section{What do the new findings imply?}

- Menstrual health research and practice must increase attention to menstrual pain, heavy bleeding and the quality of care provided by healthcare services.

- While women reported missing work due to menstruation, a holistic view of menstrual health is needed as many women endured distress and discomfort to remain at work.

- The discourses used to advocate for increased attention to adolescent menstrual health may be detrimental to menstrual health over the life course, reinforcing expectations on adult women's management.

Despite increasing acknowledgement of the importance of menstrual experiences for the health, well-being and social participation of 
those who menstruate over their life course, research and policy efforts have overwhelmingly focused on adolescent girls. ${ }^{1}$ Adult women's experiences, particularly in workplaces, have been neglected. ${ }^{2}$ A 2019 systematic review of qualitative studies of women's and girls' experiences of menstruation in low-income and middle-income countries found only 12 of 76 included studies focused on adult women. ${ }^{3}$ Of these, only one medium-quality study and two low-quality studies included any mention of women's workplace experiences.

Past qualitative studies focused on women's experiences at home have identified difficulties in accessing comfortable and reliable materials, supportive sanitation infrastructure, mechanisms for menstrual waste disposal and social support, as well as challenges due to restrictive societal expectations and menstrual stigma. ${ }^{4-7}$ While experiences at home are important, research with adolescents has found greater difficulties managing menstruation at school and we hypothesise that adult women also face greater difficulties while at work. ${ }^{8}$

Equal opportunity for participation in safe and decent work is essential for women's empowerment and gender equality. ${ }^{9}$ Paid work can provide opportunities for improved living standards, autonomy and health for women and their families. However, there remain a plethora of gendered barriers to equal work, and workplaces are often sites of compromised human rights, health and well-being. ${ }^{10}$ Menstruation represents an overlooked gendered workplace experience.

This study provides the first exploration of women's menstrual experiences at work in Uganda. Guided by a grounded theory approach, we aimed to develop a nuanced understanding of women's menstrual experiences, contributors to experience and impacts on health, well-being and participation at work. Our research focusses on three dominant workplaces for women ${ }^{11}$ : markets, government primary schools and public healthcare facilities (HCFs).

\section{METHODS}

This study forms part of a larger equal status, sequential exploratory, mixed-methods research programme investigating the sanitation and menstrual experiences of women working in Mukono District, Uganda. Given the paucity of research on this topic, we drew on a grounded theory approach for the qualitative portion and sought to inductively develop mid-level theory to describe women's experiences. ${ }^{12} 13$ The study is reported according to Standards for Reporting Qualitative Research (SRQR) guidance $^{14}$ (online supplemental materials 1 ).

\section{Setting and site selection}

Mukono District is part of the central region of Uganda. It has a population of over 600000 , of which approximately $33 \%$ reside in urban areas and $43 \%$ rely on subsistence farming. ${ }^{15}$ Of women aged 18 and over, illiteracy was estimated at $20 \%$ in $2014 .{ }^{15}$ The district was selected as a populous and emerging industrial area, exhibiting both rural and township characteristics. The local government assisted in identifying all district marketplaces, along with nearby HCFs and schools. Markets were considered eligible for inclusion if they operated a minimum of 8 hours per day for at least 3 days per week. Ten markets were identified. Those in close geographical proximity were considered sections of a single market area.

For qualitative data collection, a subset of five markets were purposefully selected based on: size, goods sold and subcounty area. We selected the large central market in Mukono municipality (estimated 1200 female workers), along with four smaller markets (estimated 25-100 female workers). Five government HCFs and five primary day schools in closest proximity to the markets were included.

\section{Participants}

Women aged 18-49, who had worked in their workplace at least 3 days per week over the last month, and who had menstruated in the past 3 months were eligible to participate. Participants were initially purposively sampled using a matrix to achieve variability across age group, tenure at the workplace, the number of days worked and type of goods sold or role at the HCF. Our theoretical sampling was limited by feasibility and the time available to access markets. However, concurrent analysis informed continued sampling for diversity across age groups and the selling of food and non-food items in markets; further, we noted variability in the extent to which menstruation impacted working days and the types of sanitation facilities being used by participants and did not pursue additional sampling on these characteristics.

\section{Data collection}

Thirty-five semistructured in-depth interviews (IDIs) were undertaken in February 2020 and lasted 45-60 min of discussion. Three experienced, female interviewers fluent in Luganda (the main spoken language) and English received 4 days of training, including a pilot interview in a nearby Wakiso District market. Interviews were conducted in Luganda or English depending on participants' preferences. Women in the selected workplaces were approached and screened for eligibility and consent. Interviews were conducted onsite in workplaces with auditory and, where possible, visual privacy. Particularly in markets, interviews were paused as needed to ensure privacy and minimise disruption to women's work tasks. The consent process highlighted that interviews would discuss participant experiences of sanitation and menstruation in the workplace. In a subset of markets, some women declined participation as they were unwilling to be audio-recorded.

The topic guide evolved throughout data collection. IDIs commenced with an exploration of the participant's typical workday schedule, followed by the sanitation portion of the interview seeking to understand experiences of urination and defecation, perspectives on 
workplace facilities, and their influences on work life. The menstrual portion of the topic guide included: narrative of experiencing the last menstruation; managing menstruation at work including material use, changing and disposal and washing the body; experience of menstrual pain and symptoms; and experience of menstruation at work including contrasting period and non-period days, expected behavioural changes and perceived impacts on work life. All participants were asked for recommendations for government to improve women's sanitation and menstrual experience to conclude the interview.

Interviewers transcribed and translated their own recordings. Information about tone, expression and pauses were included in transcripts, as were gestures and other descriptions recalled by interviewers. Luganda was included in the English transcripts where there were multiple translation options or terms held meaning beyond the English translation.

\section{Analysis}

Analysis commenced concurrently with data collection. Daily debriefings were undertaken. Each interviewer's first interview was transcribed and used for preliminary line by line coding by JH and SPSK, additional training, and further review of the topic guide. A second transcript was completed after interviewers' seventh interview and coding undertaken. At this time, a full study team meeting identified emerging concepts, and developed a preliminary core category. Modifications to the topic guide were made to explore emerging concepts, including asking participants to compare their sanitation and menstrual-related stressors to other workplace challenges and discussing changes in experience since they started work at their current workplace.

After all transcripts were completed, analysis followed phases of open, axial and selective coding ${ }^{16}{ }^{17}$ to facilitate constant comparison. Open coding was conducted for the 21 marketplace interviews using NVivo V.12 (JH) and memos generated for each participant and workplace. We then undertook axial coding to link similar codes, identify relationships between codes, and further develop the core category. This was shared through meetings with JH, SPSK and JB, and compared against notes from debriefing sessions. SPSK coded six transcripts and found agreement on emergent categories and the core category. Interviews with women in schools and HCFs were then coded, and memos generated. A selective coding approach was taken, with transcripts coded against all emerging categories, not only the core category, facilitating contrast of these experiences with those in markets, testing the emerging theory, and refining categories. Finally, interviews in marketplaces were recoded by $\mathrm{JH}$ against the final conceptual model, with attention to divergent cases. The research plan included participant validation; however, we were unable to undertake planned follow-up focus group discussions due to the COVID-19 pandemic restrictions on travel outside the home and meeting in groups.

\section{Reflexivity}

This study is a collaboration between Ugandan and foreign investigators. During training, the study team documented the perspectives they brought to the work, with these perspectives revisited during debriefing and analysis sessions to promote continued reflexivity. $\mathrm{JH}$ led the analysis as a foreign woman not fluent in Luganda, with past research on menstrual experiences. In particular, JH had led a metasynthesis of qualitative studies of menstrual health, developing an integrated model of menstrual experience. ${ }^{3}$ While the analysis for this study was performed inductively, it was impossible for this team member to have delayed literature review. ${ }^{18}$ This past work also informed initial coverage of the topic guide. SPSK and JB guided interpretation of emergent categories as a male and female Ugandan, respectively, with limited past menstrual health research, and SPSK undertook independent coding for validation. The three female interviewers had past experience conducting interviews and surveys on reproductive health topics but had not previously worked on studies focused on menstrual health. During training, a brief overview of key topics in past menstrual health research was included. We recognise that this manuscript is written for local and foreign audiences. ${ }^{19}$ We aim to inform local policy and international approaches to menstrual health.

\section{Patient and public involvement}

We were unable to undertake planned participant involvement in shaping response to these findings due to the COVID-19 pandemic restrictions.

\section{RESULTS}

Participant characteristics are displayed in table 1. Of the 21 participants from markets, 7 worked in the large central market and the remainder across the 4 smaller markets. Teachers and HCF workers represented five different schools and HCFs.

Figure 1 presents a conceptual map of the categories identified to explain women's menstrual experiences at work. 'Being a responsible woman' emerged as the core category and substantive theory underpinning experiences. The conceptual map and identified categories were consistent across worker groups; differences in manifestation are noted in the description of each category.

Quotes are reported alongside participant identifier (ID), workplace type and participant age. Workplace IDs are provided for markets (A-E), but not for HCFs and schools to protect participant anonymity. Additional quotations are included as online supplemental materials 2 .

\section{Being a responsible woman}

Participants viewed menstruation as a natural part of female life that each woman must navigate responsibly. Although a natural process, menstruation was considered dirty and shameful. Thus, acting as a responsible woman meant keeping oneself clean and menstruation secret. 


\begin{tabular}{|c|c|c|}
\hline & $\mathbf{n}$ & $\%$ \\
\hline \multicolumn{3}{|l|}{ Workplace } \\
\hline Market & 21 & 60.0 \\
\hline Sells produce & 6 & \\
\hline Sells cooked food & 4 & \\
\hline Sells goods & 9 & \\
\hline Sells services (eg, salon) & 2 & \\
\hline School & 7 & 20.0 \\
\hline Teacher & 7 & \\
\hline Healthcare facility & 7 & 20.0 \\
\hline Clinical staff & 5 & \\
\hline Non-clinical staff & 2 & \\
\hline \multicolumn{3}{|l|}{ Age } \\
\hline $18-24$ & 9 & 25.7 \\
\hline $25-29$ & 4 & 11.4 \\
\hline 30-39 & 14 & 40.0 \\
\hline $40-49$ & 8 & 22.9 \\
\hline \multicolumn{3}{|l|}{ Tenure at workplace } \\
\hline$<1$ year & 7 & 20.0 \\
\hline $1-4$ years & 20 & 57.1 \\
\hline $5+$ years & 8 & 22.8 \\
\hline \multicolumn{3}{|l|}{ Usual days worked per week } \\
\hline $3-5$ days & 11 & 31.5 \\
\hline 6 days & 13 & 37.1 \\
\hline 7 days & 11 & 31.4 \\
\hline Hours worked in a typical day & Mean & SD \\
\hline Market & 12.2 & 1.7 \\
\hline School & 11.9 & 1.6 \\
\hline Healthcare facility & 9.7 & 2.0 \\
\hline
\end{tabular}

on with usual activities during menstruation represented a failure of womanhood.

What would I have done? I do what a woman should do... I don't take anything, but I pad myself very well then I sleep... Then I have a feeling too that how can I be seen as an adult? How will it be if I map [soil outer garments]? So as an adult, I have to time myself then I run to change. (ID-21, Market-D, 49)

By the time one starts periods she is regarded as an adult, but you find when there are clots of blood in the bathroom, you go to squat and see that. Do you understand? That I wouldn't expect one to do it. (ID-23, Market-D, 20)

Women who failed to uphold perceived hygiene and secrecy standards were met with disgust and shame. Exceptions were made for women with severe menstrual pain or heavy bleeding; these afflictions were viewed as medical concerns in need of compassion, advice and some leniency.

I want to continue telling the women the way they are supposed to behave during their menstrual periods, they have to keep clean as women... (ID-27, HCF, 24)

These deeply held expectations of womanly conduct permeated every aspect of menstrual experience. Although the workplace infrastructure and resources presented barriers or facilitators to caring for the body during menstruation, women were expected to uphold the prescribed standards regardless of circumstance.

... I can't tell my boss that I have failed to work because of my period. That doesn't help him. (ID-8, Market-A, 28)

you just encourage yourself, then you go and do the work. You can't dodge working because of that. (ID-12, Teacher, 41)

\section{Keeping clean}

Menstruation, and by extension a menstruating woman, was viewed as unclean. Failures to uphold required cleanliness were associated with intense disgust ('okwenyinyala'), expressed using this strong Luganda term, to evoke

A responsible woman upheld these ideals without assistance. Struggles with menstruation were viewed as the purview of adolescent girls. Failing to manage and carry

\section{BEING A RESPONSIBLE WOMAN}

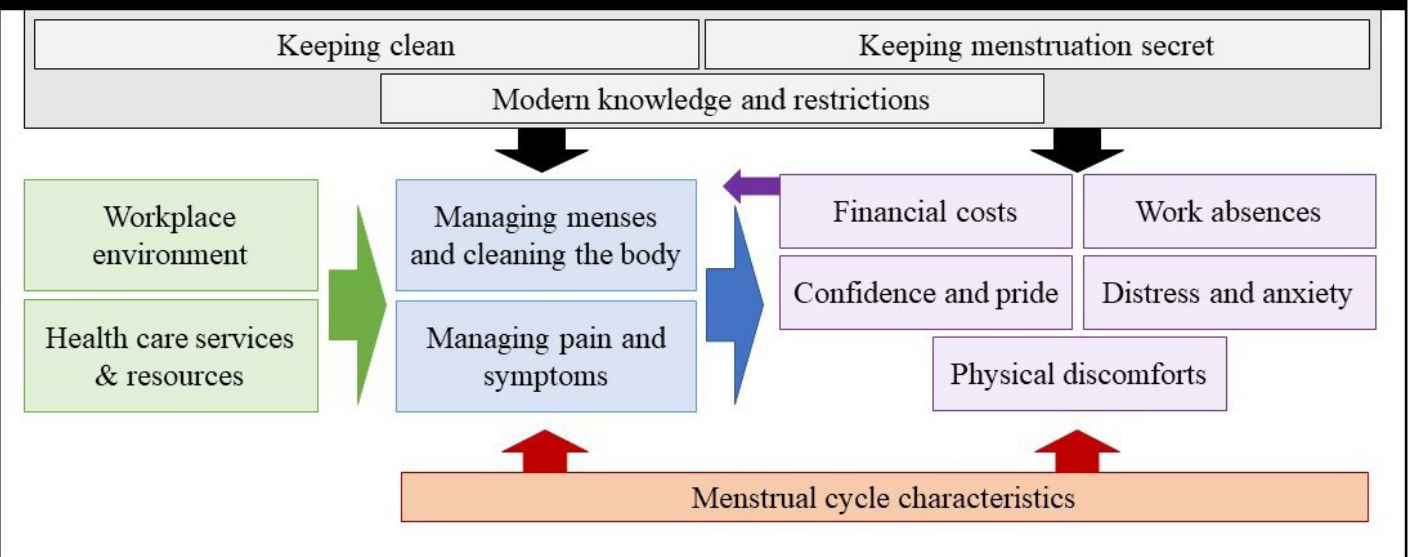

Figure 1 Visual representation of identified categories and emergent theory. 
a visceral disgust to something such as that producing a stench.

Women expressed personalised, authoritative views about what it meant to maintain hygiene during menstruation. These standards shaped her responses to the workplace facilities and her strategies for menstrual management.

It shows someone's personal hygiene, so once you map [soil outer garments] then you are regarded unhygienic. (ID-21, Market-D, 49)

We have to admit that we are dirty during that period. There are women who don't know how to clean, for example when someone goes behind [to change their materials] yes, they go with bottles of water but some don't do any other thing after that. (ID-20, Market-C, 30)

Hygiene behaviours were framed as both responsible, and essential to 'feel fresh' and comfortable at work. To be considered clean, the body needed to be free from odour or visible menstrual blood, and sanitation and bathing facilities should be free from blood or disposed menstrual materials.

... you have to clean yourself and change, then you will be comfortable in class. (ID-11, Teacher, 25)

\section{Keeping menstruation secret}

Menstruation was viewed as a deeply personal women's issue that should not be discussed with others. Women used coded language to refer to menstruation, ranging from using the English "periods", "p's" or "that time", to codified terms in Luganda such as "in red", "being up" ["oli waggulu"/ "munnange ndi waggulu"], or "my days" [ "ennaku zange"]. Expectations of secrecy overlapped with requirements for cleanliness, such that others should not see blood or materials in sanitation facilities or when materials were washed and dried.

They do not spread them outside, a woman does not spread those private towels outside, and they are put inside the house. (ID-28, Market-E, 37)

for me when I see people talking about it, I feel disgusted and wonder why they can't discuss about other things. (ID25, Teacher, 35)

It was considered particularly inappropriate for men to be exposed to blood or discussion about menstruation. If required, menstruation could be discussed with trusted women. These conversations were shaped by the expectation that a responsible woman should be capable of managing her menses alone. However, if a woman was seen to have soiled her outer garments, it was the responsibility of nearby women to alert her and provide her with a cloth to cover herself.

Those experiencing pain or heavy bleeding were excepted from strict expectations to keep menstruation secret. They were viewed as needing to seek advice and support from other women and healthcare providers. The few women who described themselves as freer in their discussion of menstruation knew that this was a violation of the social norm and viewed themselves as either a positive deviant or an exception due to struggles with pain or heavy bleeding. Some teachers and HCF workers expressed less restrictive attitudes, although sharing was always limited to close female friends. For this group, teaching students or patients about menstruation was excepted from required secrecy, and distinct from the shame associated with sharing personal experiences.

Most women share when they want solutions to their issues for example those who use family [contraception], they come and tell you that ever since I got the family planning injection I bleed endlessly. With that one it is understandable because she is looking for advice... (ID-20, Market-C, 30)

\section{Modern knowledge and restrictions Knowledge}

Women spoke authoritatively about menstrual anatomy, management and hygiene, influencing their practices and anxiety during menstruation. However, they held varied views on whether genitals should be washed with soap, and on the responsible way to dispose of pads. When noting that sex should be avoided during menstruation, women described that menstrual blood could be forced back up into the body and cause illness. Despite a medical view of menstrual pain, no participants discussed medical reasons or physiology underlying pain or symptoms. Most believed they were likely to contract reproductive tract infections from sanitation facilities, four stated they were more vulnerable during menstruation.

\section{Restrictions}

Participants identified as modern, urban, working women and expressed that restrictions on menstruating women were outdated. Some reflected that they had feared taboos when they were younger, but later believed these were exaggerations to teach them appropriate menstrual care. All women stated that sex during menstruation was inappropriate. For Muslim women, religious restrictions required they avoid prayer and fasting during menses. Two women expressed concerns about the use of disposed menstrual materials for witchcraft, and one reported avoiding cooking food for others while menstruating. Three women described that customers held taboos or would be disgusted by a menstruating woman so restricted their behaviour despite not endorsing this belief.

I sell food, a customer after knowing that you are in that period...some customers feel disgusted... you can't be in your period and serve them food. (ID-29, Market-E 26)

\section{Workplace environment}

Physical and interpersonal characteristics of workplaces influenced women's experience.

Water, sanitation and hygiene services

Workplaces had improved pit latrines or pour/flush toilets. Women strategised about their sanitation use during menstruation, balancing the distance to facilities, 
availability of water, cleanliness, privacy and cost. For example, one HCF worker described using the nearby pit latrine for urination but journeying to the toilet in the maternity care area for menstrual management:

the toilet is comfortable even if I need to change and wash some part, I can be able to access water, I can be able to access a basin but if I use this one [the nearer pit latrine], there are no provisions for that. (ID-33, HCF, 34).

While a market woman travelled to a nearby church during menstruation:

I like their doors, they are strong, and I can be safe because I am able to close myself in. They are metal doors, the place is safe. (ID-8, Market-A, 28).

Five women working in markets stated that they required more frequent visits to the sanitation facility during menstruation. In four of five markets, users had to pay per use, so this incurred increased costs. Many teachers and HCF workers lived in quarters close to their workplaces, so could more easily travel home for menstrual management.

Haaa, for me when I am in my periods, I don't want to use that thing there [school facility] (laughs). I have to go, that is the time I go home almost all the time as long as I don't have a lesson because it is easy to get water from there. (ID-

14, Teacher, 21)

In all markets, at least one sanitation facility had an attached bathroom, although in two (Market-D, Market-E) no participants used this space due to cleanliness and privacy concerns. Almost all participants believed it was essential for hygiene to wash the genitals each time menstrual materials were changed. Thus, the availability of water and cost of using the bathroom for washing were important.

I may go there three times in a day [the paid sanitation facility during menstruation] and you must pay so the expense is high, but you must clean up to maintain proper hygiene. (ID-6, Market-A, 23)

Available disposal facilities for menstrual waste shaped women's practices. In pour/flush toilets workplaces often provided buckets for disposal. These were absent in pit latrines and women disposed into the latrine.

They should also clean the bin where we dispose the pads because it being dirty forces people to throw in the toilet and this blocks it. (ID-29, Market-E, 26)

Some teachers and HCF workers used menstrual materials from their workplace to offset the cost of commercial products or if they were caught without materials. Three HCF workers described using cotton and gauze from the facility and two teachers used pads or cotton purchased using school funds for teachers and students.

\section{Social environment}

Access to supportive colleagues shaped women's work lives. In markets, those without coworkers or good relationships with nearby vendors worried that they would miss customers, or goods would be tampered when they left their work site. For teachers, those who could shift work burden to colleagues when experiencing illness (including menstrual pain) experienced relief. In HCFs, women working alone or on overnight shifts had limited assistance.

like when I am in my menses, I tell my colleague that you be taking the weight [of clients]... (ID-10, HCF, 38)

Yeah, on the first day [of menstruation] I come to work late, I come like at $10 \mathrm{am}$ or $11 \mathrm{am}$. I tell my sister to come and sit in for me. (ID-32, Market-E, 21)

Rules to dissuade women from disposing of materials into flush toilets influenced practices. In one market women described that announcements were made over the workplace radio, while in another, signs indicated a fine.

In one Muslim-founded school, religious cleansing practices meant that water was always available at the sanitation facility, benefiting all women during menstruation.

\section{Health care services and resources}

Access to care and resources such as pain relief medication critically contributed to menstrual experiences at work. Eighteen women had sought healthcare advice regarding menstrual pain, irregular or heavy bleeding or a perceived genital infection related to their menstruation. Women reported variable success with health services; most were told their experience was normal and received a painkiller, which was variably successful. One received scans for irregular or painful periods; none reported a diagnosis or greater insight into their symptoms. Two women reported falling severely ill each period, including fevers and nausea. They reported no success with healthcare, but that difficulties resolved after their first birth.

\section{Menstrual cycle characteristics}

The level of pain, volume of bleeding and other symptoms accompanying menstruation varied and were a central part of women's experiences. Women described themselves as fortunate or unfortunate depending on their symptoms. Higher degrees of pain caused greater distress and disruption at work.

I feel weak, yet I have to work remember, it is flowing, once you stand up, it pours heavily, then you say, "ooh God this is war!", you are just praying for the three days to come to an end. (ID-19, Market-D, 24)

Heavy bleeding was frequently reported and resulted in anxiety about one's health, increased management challenges, and fears of soiling. Unpredictable periods were a source of anxiety as women felt they may unexpectedly soil their outer garments.

Eleven women described themselves as irritable during menstruation. Mood changes impacted their interactions with customers, colleagues, students or patients. At times women invoked their menstrual pain or discomfort as 
sources of frustration, for others these were an independent emotional change.

Yes I become rude, I am very angry, I find myself not very happy, so I find myself being rude till I finish my periods [laughter] but now that I know I try to control (ID-33, HCF, 34)

Many women reported feeling weak or lacking energy during menstruation, which also impacted their workday.

\section{Managing pain and symptoms}

Menstruating at work meant managing pain and symptoms, with women's comfort and participation dependent on the extent to which they were able to minimise pain. Many used paracetamol and ibuprofen, while others believed that it was unhealthy to take pain killers. Some reported restricting medication use, fearing side-effects or believing they would become reliant. Women also used hot water, tea and herbal remedies.

With cramps, I have to take Ibuprofen. Like I take them when I am coming for day duty, I can take them at around 5 [am] such that they start working. When I am on day duty, I am fine but when I delay to take meals, it disturbs me. (ID-1, HCF, 23)

Ok I used to take some Panadol but once I was on radio and they said that it was not good to take Panadol and now I don't take anything... I used to take it a lot. (ID-31, Teacher, 42)

\section{Managing menses and cleaning the body Being prepared}

Many women described the importance of being well equipped' or 'prepared'. Almost all stored menstrual materials in their home for when menstruation started. Distress occurred if they were caught unprepared.

I am aware of my days, I was prepared then. I had my pads ready... (ID-8, Market-A, 28)

To further protect themselves from surprises and accompanying shame, women strategically selected clothing. They wore dark colours, flowing dresses and tight shorts over their underwear for added protection and to disguise potential leaks.

I confine myself and I don't put on short clothes, light clothes, I put on black clothes and because I bleed a lot, I can map [soil] any time. If you are in black, it is not easy to notice that you have mapped [soiled]. (ID-26, Market-D, 39)

\section{Selecting materials}

Women described at length reasons for selecting their menstrual materials. Participants prioritised different concerns including perceived quality, comfort, cost and availability. The majority preferred disposable pads: reported to efficiently absorb blood, not stick to the body, had adhesive to keep them in place and did not need to be washed or dried. Women who had transitioned to pads after using cloth reported improvements in their quality of life.

They really helped us. But those things we used before, the pieces of cloth really disturbed people, there was no security at all... (ID-24, School, 48)

They are expensive, but you have to use them because they are easy and worth using. (ID-2, Market-B, 35)

Almost every woman using pads reported that cheaper brands were ineffective, caused irritation or contained harmful chemicals. Thus, they purchased higher priced products. A few avoided pads entirely due to concerns about quality, soiling, harmful effects or did not see the value.

I fear pads, I didn't ever go for them, I feared pads from long ago, same applies to children's diapers, I never give my children diapers. (ID-28, Market-E, 37)

Some younger participants stated that disposable pads were the only acceptable menstrual material, so one had to find or borrow money with no alternative. Other women supplemented pad use with cloth or cheaper pads, conserving their preferred materials for work.

If am at home I use my pieces of cloth because I may clean as much as possible, yet it is not the case at work. While at home I can check as many times as I need to check which is not possible if am at work because there is no room for that. (ID-6, Market-A, 23)

A few women used commercially produced reusable pads and found these to alleviate disposal concerns, offer comfort and cost savings. They were washed and dried discreetly at home. Others had not heard of or tried these products, and a few had past negative experiences.

I do walk a lot and with the washable pad I am comfortable because it does not irritate me. I spend the whole day without any problem. That is the beauty with that pad. Some ladies find a problem with washing but for me I find no problem with this particular pad. (ID-20, Market-C, 30)

Women reported wanting to know more about the properties of different materials to inform their choices.

\section{Changing materials}

Participants changed their materials at varied frequencies to avoid soiling, odour and discomfort, and to conserve pads. Around half used the workplace sanitation facility or attached bathroom to change their materials. Some felt comfortable, while others were uneasy but felt there was no other choice. Many women described that facilities were too dirty, costly or insufficiently private for changing and used alternatives at nearby businesses or travelled home. Women with stores reported changing there, and one woman hid underneath her market stall. Some women using reusables travelled home so they could immediately wash and dry these; others rinsed at work or transported home later in a bag for cleaning.

...the problem I have is the bad odour when I am in my periods. I have to keep changing so that I do not smell for 
my neighbours here at the stall, otherwise one pad would have been enough for me to go through the day. (ID-4, Market-A, 43)

I can easily change because behind here [her store] I am alone... no one can see you. (ID-16, Market-C, 18)

\section{Disposal}

Women reported a variety of disposal strategies depending on their preferences and habits. Many disposed into pit latrines. A vocal subset believed this resulted in latrines filling too quickly and bemoaned this practice. In workplaces with toilets and bins, most participants reported compliance and disposed into the bin, although often complained that these were full and that other women's used pads were visible or clogged in the toilet. Some women carried their materials home to burn them or dispose into their home pit latrine, fearing they would be seen by others.

\section{Washing the body}

For almost all women, keeping clean required washing the genitals each time menstrual materials were changed, with many preferring to wash the whole body. Even when women were able to change their materials in the workplace, they were frequently dissatisfied with the irregular availability of water for washing the genitals and body. More often this was the most significant stressor for managing menstruation and motivated many to travel home or to other facilities.

\begin{abstract}
My dear can you change and fail to wash up? No, it is impossible! At least I wash my private parts then change my knickers and pad. We live near, but those who don't reside near here are the ones who find it hard, they just have to remove the used one and wear another pad. (ID-34, School, 48)

... when am in my periods that I may need to be extra clean but when am off my periods then am not so much concerned about going to bathe during the day. (ID-33, HCF, 34)
\end{abstract}

\section{Confidence and pride}

Women expressed a sense of confidence and pride in taking responsible care of themselves and 'getting on with things' during their period. This confidence did not always mean an absence of harms for their workday. For women, maintaining cleanliness and secrecy was paramount, and other consequences described below were often not framed as harms of menstruation.

I always ensure I have taken very good care of myself. I make sure my pad is firm, so that as I move about, I feel confident. I always put on a tight short so that in case of anything...it cannot drop. (ID-13, Market-C, 37)

Yes [feels comfortable at work], because I know I am safe, I am not going to map because that is what people worry about but for me, I know I am safe I have like 3 pads in my bag for emergencies, I often check myself to see (ID-8, Market-A, 28)

\section{Distress and anxiety}

Women vigilantly monitored their cleanliness and the risk of soiling during menstruation. The extent of worry varied, depending on the confidence they held in management behaviours, the presence of pain or heavy bleeding, the workplace resources, and women's social supports. Experiences ranged from constant anxiety while at work, to women who felt 'free and easy'.

I carefully sit and I check myself all the time, you never know it can pass through (ID-29, Market-E, 26)

Obviously you cannot have peace because every time you are worried. (ID-35, HCF, 35)

Women employed different strategies to mitigate anxiety; six participants described withdrawing from others during menstruation.

\section{Financial costs}

Menstruating incurred costs, imposing financial stress. For some women, pads were affordable, for others, expensive but worthwhile. Still others found them too costly, mitigating expenses with other materials. This was not restricted to women working in markets, teachers and HCF workers also described cost pressures in relation to purchasing pads.

You have to give it priority and say this month I will buy this number of packets and keep them there so that when I use one, then I will know I have to replace it. (ID-34, School, 48)

What I do these days is save money and buy pads so that by the end of one period of menstruation, I am prepared for the next (ID-16, Market-C, 18)

Market women incurred costs visiting the sanitation facility or bathroom more often to change materials, as well as the potential lost income of missing customers.

\section{Physical discomfort}

For many women, menstruating at work was characterised by discomforts including enduring menstrual pain, not feeling clean and positioning the body such that the risk of soiling was minimised. Four women reported experiences of soreness or itching in their genitals. Ten described fatigue, desiring more rest or reduced tasks at work, but needing to persist.

Yes, because when I am not in my periods, I am able to move freely and perform my activities very well. Another thing is, I don't feel well when in my period. They are only 3 days but they seem like a month (ID-8, Market-A, 28)

...the way you found me here seated? No, I limit myself. I only sit when I have just padded myself then after thirty minutes to one hour I stand up. I don't need stains. I can't stand that embarrassment... (ID-18, Market-C, 39)

\section{Work absences}

Many women reported that they missed or reduced work during menstruation due to pain, concerns about soiling or inadequate sanitation facilities. Many rejected 
the assertion that menstruation could impact their work, feeling that this implied they were failing to manage responsibly. At the same time, women described clear examples of time lost, such as travelling home to change materials or enduring significant discomfort to remain at work, with impacts on their performance.

No for me, when I get to know that I am menstruating, because it is much [heavy bleeding] and our toilets are also dirty, I don't come [to work] until I am done with menstruating. (ID-5, Market-A, 27)

Yes, like today it was too much, I reached at work at around 1:00 or 1:30 PM...Yes, until the pain decreased and I came. (ID-7, Market-A, 40)

even if you are in class you're uncomfortable and you are there like a statue but you can't work when the pain is too much, especially on the first day. You feel the headache, the back is as if you have been cut by swords and the abdominal pain... and in fact you are there but you feel you are not present just because of the pain you are feeling. (ID-31, Teacher, 42)

Some women suggested that they would prefer not to be working during their period but had little choice due to workplace expectations or because they could not afford the lost income.

They used to say a person menstruating doesn't work, doesn't do what, doesn't do this, but today if you sit at home, what will you eat? You also have rent to pay. (ID-19, Market-D, 24)

Women who reported lighter, less painful periods, consistent access to preferred products and satisfaction with the workplace environment reported fewer impacts on their workday.

I keep on going because it is natural and there is no way you can avoid it so you be free every day and you're used to it, that it happens in every month, so you just have to concentrate. (ID-30, Market-E, 41)

\section{DISCUSSION}

Women working across markets, schools and HCFs in Mukono District experienced significant consequences for their work, finances and health due to menstruation. A combination of unsupportive sanitation infrastructure, anxieties about soiling and pain contributed to work absences, consistent with research among adolescents. ${ }^{3} 20$ However, absenteeism was not the most common consequence of unmet menstrual needs and a holistic view of menstrual health is needed to support decent working conditions for women. Women described missing parts of the workday or reducing their engagement, while many others endured pain, discomfort, anxiety and fatigue to remain at work. Menstruation was not considered an acceptable reason for absence for women employed by others, while self-employed market women could not afford to miss sales. The need to look beyond absenteeism to the health and well-being consequences of unmet menstrual needs has been emphasised for adolescents. ${ }^{21}$ Our findings extend this call to adult women, highlighting that presence at work may be a poor indicator for menstrual health if women's physical and mental wellbeing is unsupported.

The need to act as a responsible woman underpinned women's menstrual experience. Secrecy and cleanliness were the highest priorities. From the authors' perspective, the stringency of these expectations resulted in harms for women. Heightened anxiety at the prospect of exposure, feelings of shame and disgust, and the financial and time burden of extensive body washing, resulted in consequences for health and work. This core category echoes decades of research from high-income country contexts, where feminist scholars have identified 'menstrual etiquette' as a restrictive gender norm governing women's bodies. ${ }^{22-24}$ However, from participants' perspectives, this was merely a requirement of being a woman. They felt pride in successfully enacting menstrual requirements and judged other women for failures. Some recommended government improvements focus on teaching other women to better uphold these ideals.

Our findings suggest that any advocacy or intervention seeking to improve menstrual health needs must navigate this norm. Notably, participants released adolescent girls from stringent menstrual responsibilities. As energy for improving menstrual health gains traction in Uganda, our findings suggest that focus on assisting adolescent girls must be cautious not to exacerbate the pressures placed on adult women. Conversely, population-wide efforts to reduce menstrual stigma over the life course may benefit adult women, as may greater acknowledgement of menstrual-related infrastructure and resource needs. However, advocates must be aware that suggesting adult women struggle during menstruation may be met with resistance as this statement confers judgement, and priorities to destigmatise menstruation may conflict with women's own construction of menstruation.

Our results show that more attention is needed to the quality of disposable pads being sold . Women reported cheaper brands caused burning, irritation, discomfort and leakage, but that the higher performing products were a financial burden. Women sought to be informed consumers of menstrual products and desired more information about their options. Supporting the affordability of a range of product choices for women would improve menstrual experiences and alleviate some financial burden.

Unsupportive workplace water and sanitation infrastructure were challenging. Many women believed that it was essential to wash their genitals and body when changing materials. Water availability at sanitation facilities, and the cleanliness, privacy and cost of bathrooms often failed to support this practice, placing additional stress on women. Many women's recommendations for improvement focused on addressing this environment. Intervention approaches which provide education 
around genital washing, support preferred practices in clean, affordable facilities and minimise exposure to dirty water are indicated.

Pain, heavy bleeding and other symptoms associated with menstruation were key parts of women's experience. Menstrual health advocacy has frequently focused on the management of menstrual bleeding. ${ }^{25}$ Women in our study considered symptoms associated with menstruation as equally relevant to menstrual health. Indeed, physical symptoms and menstrual management were often interlinked, for example, heavy bleeding had significant implications for menstrual material use and changes. Symptoms, disorders and pain require greater attention in menstrual health research and practice.

The variable, but often limited, support received from healthcare services, and women's knowledge of menstrual disorders and pain relief options require further exploration. Future research should prioritise understanding women's knowledge, alongside healthcare provider knowledge, and the quality of care in health services. Our findings indicate women need greater support. We also found that they approach healthcare services for menstrual needs, indicating these services as an avenue for intervention delivery. Educational programmes on pain and symptoms would be acceptable for this population, as these challenges were excepted from required secrecy around menstruation. Further, additional knowledge gaps including the transmission and prevention of reproductive tract infections, hygienic genital care and menstrual anatomy were identified through our study.

\section{Strengths and limitations}

To the best of our knowledge, this study is the first to investigate women's menstrual experiences at work in Uganda. Although we were informed by past research, ${ }^{34}$ we took an inductive approach to analysis and prioritised understanding participant experiences. In our application of a grounded theory approach, we took a pragmatic stance, attending to women's constructions of their lived experience as well as factors identified as impacting experiences. Feasibility constraints limited the extent of theoretical sampling of participants, which was a major limitation of this study and our development of grounded theory. Through concurrent data collection and analysis, we monitored the diversity of experiences captured, and modifications to the topic guide were made to explore emergent categories. Women's concerns around audio recording in some markets meant the perspectives of these women were missed. The total sample size was consistent with our a-priori specification. No new categories emerged in coding the interviews with teachers and HCF workers against the emergent categories based on interviews with women working in markets, although these brought additional depth. Diverging experiences are highlighted in the described results but did not alter the theoretical categories. We believe we achieved inductive thematic saturation. ${ }^{26}$ Unfortunately, due to the COVID-19 pandemic we were unable to undertake planned participant validation activities. Further follow-up with women should be pursued in future studies and insights from workplace administrators would assist in identifying workplace changes.

\section{CONCLUSIONS}

Workplaces are crucial environments to support women's health and economic empowerment. Upholding gendered ideals of responsible menstrual management places women under pressure to keep clean and menstruation hidden. Women's menstrual characteristics such as their level of pain, access to healthcare resources and the workplace environment impacted experiences of upholding these expectations and women's ability to care for their body at work. Together, this shaped the influence of menstruation on women's life at work, including feelings of confidence and pride, distress and anxiety, physical discomforts, financial costs and work absences. Women's menstrual health in the workplace requires more attention from researchers, public and private sectors to support equal opportunities for decent work and women's health.

\section{Author affiliations}

${ }^{1}$ The Water Institute, Department of Environmental Health and Engineering, Johns Hopkins University Bloomberg School of Public Health, Baltimore, Maryland, USA ${ }^{2}$ Department of Community Health and Behavioural Sciences, Makerere University College of Health Sciences, Kampala, Uganda

${ }^{3}$ Department of Epidemiology \& Biostatistics, Makerere University College of Health Sciences, Kampala, Uganda

Twitter Julie Hennegan @julie_hennegan and Natalie G Exum @NatalieGExum

Acknowledgements The authors are grateful to the women who participated in the study and shared their experiences. They thank their skilled qualitative interviewing team: Brenda Nakazibwe, Prossie Aliwebwa, and Resty Nakayima, and project coordinator Petranilla Nakamya.

Contributors JH: Conceptualisation; methodology; formal analysis; investigation; data curation; writing - original draft; visualisation; supervision; project administration. SPSK: Methodology; formal analysis; investigation; validation; writing - reviewing \& editing; supervision; project administration. NGE: Methodology; validation; writing - reviewing \& editing; project administration. KJS: Conceptualisation; validation; resources; writing - review \& editing; supervision; project administration; funding acquisition. FEM: Methodology; validation; writing - review \& editing; supervision. JB: Methodology; formal analysis; investigation; validation; writing - reviewing \& editing; supervision. All authors have approved the final manuscript.

Funding This study was made possible by the 0sprey Foundation of Maryland.

\section{Competing interests None declared.}

\section{Patient consent for publication Not required.}

Ethics approval All participants provided written informed consent, including for audio recording, prior to interview. All interviews were conducted with auditory, and where possible, visual privacy. Participants were informed of their right not to discuss topics and to decline to answer any questions or end the interview. Workplace administrators (Headteachers, Health Care Facility Administrators and Market Chairpersons) permitted recruitment of participants from their workplaces. Approval for the study in the area was also provided by the Mukono district chief administrator's office and the Mukono Municipality Town clerk's office. Ethical approval was provided by Makerere University School of Public Health Higher Degrees, Research and Ethics Committee (HDREC: 739) and Johns Hopkins Bloomberg School of Public Health Institutional Review Board (IRB: 00010015). The Uganda National Council for Science and Technology (UNCST) approved the study (ref: SS 5143)

Provenance and peer review Not commissioned; externally peer reviewed. 
Data availability statement This is a qualitative study and therefore the data generated are not suitable for sharing beyond that contained within the report and supplementary materials. Further information can be obtained from the corresponding author.

Supplemental material This content has been supplied by the author(s). It has not been vetted by BMJ Publishing Group Limited (BMJ) and may not have been peer-reviewed. Any opinions or recommendations discussed are solely those of the author(s) and are not endorsed by BMJ. BMJ disclaims all liability and responsibility arising from any reliance placed on the content. Where the content includes any translated material, BMJ does not warrant the accuracy and reliability of the translations (including but not limited to local regulations, clinical guidelines, terminology, drug names and drug dosages), and is not responsible for any error and/or omissions arising from translation and adaptation or otherwise.

Open access This is an open access article distributed in accordance with the Creative Commons Attribution Non Commercial (CC BY-NC 4.0) license, which permits others to distribute, remix, adapt, build upon this work non-commercially, and license their derivative works on different terms, provided the original work is properly cited, appropriate credit is given, any changes made indicated, and the use is non-commercial. See: http://creativecommons.org/licenses/by-nc/4.0/.

\section{ORCID iDs}

Julie Hennegan http://orcid.org/0000-0003-2011-1595

Simon P S Kibira http://orcid.org/0000-0002-7385-423X

Natalie G Exum http://orcid.org/0000-0002-8374-4034

\section{REFERENCES}

1 Bobel C. The managed body: developing girls and menstrual health in the global South. Springer, 2019.

2 Sommer M, Chandraratna S, Cavill S, et al. Managing menstruation in the workplace: an overlooked issue in low- and middle-income countries. Int J Equity Health 2016;15:86.

3 Hennegan J, Shannon AK, Rubli J, et al. Women's and girls' experiences of menstruation in low- and middle-income countries: a systematic review and qualitative metasynthesis. PLoS Med 2019;16:e1002803.

4 Scorgie F, Foster J, Stadler J, et al. "Bitten By Shyness": Menstrual Hygiene Management, Sanitation, and the Quest for Privacy in South Africa. Med Anthropol 2016;35:161-76.

5 MacRae ER, Clasen T, Dasmohapatra M, et al. 'It's like a burden on the head': redefining adequate menstrual hygiene management throughout women's varied life stages in Odisha, India. PLoS One 2019;14:e0220114.

6 Mohamed Y, Durrant K, Huggett C, et al. A qualitative exploration of menstruation-related restrictive practices in Fiji, Solomon islands and Papua New Guinea. PLoS One 2018;13:e0208224.
7 Crawford M, Menger LM, Kaufman MR. 'This is a natural process': managing menstrual stigma in Nepal. Cult Health Sex 2014;16:426-39.

8 Hennegan J, Sol L. Confidence to manage menstruation at home and at school: findings from a cross-sectional survey of schoolgirls in rural Bangladesh. Cult Health Sex 2020;22:1-20.

9 United Nations Development Programme (UNDP). Gender equality strategy 2018-2021. New York, USA: United Nations Development Programme, 2018.

10 UN Women. Transforming economies, Realizing rights. New York, United States: UN Women, 2015.

11 Kasirye I. Addressing gender gaps in the Ugandan labour market. policy Briefs 150532: economic policy research centre (EPRC, 2011.

12 Ritchie J, Lewis J, Nicholls CM, et al. Qualitative Research Practice: A guide for social science students \& researchers. 2nd Edn. London: SAGE Publications, 2014

13 Glaser B, Strauss A. The discovery of grounded theory. 1967. Weidenfield. London: Nicolson, 1967: 1-19.

14 O'Brien BC, Harris IB, Beckman TJ, et al. Standards for reporting qualitative research: a synthesis of recommendations. Acad Med 2014;89:1245-51.

15 Uganda Bureau of Statistics. The National population and housing census 2014 - area specific profile series. Kampala, Uganda: Uganda Bureau of Statistics, 2017.

16 Corbin JM, Strauss A. Grounded theory research: procedures, canons, and evaluative criteria. Qual Sociol 1990;13:3-21.

17 Corbin J, Strauss A. Basics of qualitative research: techniques and procedures for developing grounded theory. Sage publications, 2014.

18 Charmaz K, Mitchell RG. Grounded theory in ethnography. Handbook of ethnography 2001;160:174.

19 Abimbola S. The foreign gaze: authorship in academic global health. BMJ Glob Health 2019;4:e002068 p:e002068.

20 Miiro G, Rutakumwa R, Nakiyingi-Miiro J, et al. Menstrual health and school absenteeism among adolescent girls in Uganda (meniscus): a feasibility study. BMC Womens Health 2018;18:4.

21 Sommer M, Zulaika G, Schmitt M, et al. Hygiene: measuring progress for girls on menstruation; meeting report. New York \& Geneva: Columbia University and WSSCC, 2019.

22 Laws S. Issues of blood: the politics of menstruation. Springer, 1991.

23 Bobel C. New blood: Third-wave feminism and the politics of menstruation. Rutgers University Press, 2010.

24 Wootton S, Morison T. Menstrual management and the negotiation of failed Femininities: a Discursive study among low-income young women in Aotearoa (New Zealand). Womens Reprod Health 2020;7:87-106.

25 Sommer M, Caruso BA, Sahin M, et al. A time for global action: addressing girls' menstrual hygiene management needs in schools. PLoS Med 2016;13:e1001962.

26 Saunders B, Sim J, Kingstone T, et al. Saturation in qualitative research: exploring its conceptualization and operationalization. Qual Quant 2018;52:1893-907. 